\title{
An Adaptive Vector Quantization Method for Image Compression
}

\author{
A. Divya \\ Ph.D Research Scholar \\ Department of Computer Science \\ Erode Arts and Science College \\ Erode, Tamilnadu, India
}

\author{
S. Sukumaran, $\mathrm{PhD}$ \\ Associate Professor \\ Department of Computer Science \\ Erode Arts and Science College \\ Erode, Tamilnadu, India
}

\begin{abstract}
Image compression is to reduce redundancy of the image data in order to store or transmit data in an efficient form. Compression is carried out for the following reasons about reduce, the storage requirement, processing time and transmission duration. The most powerful and quantization technique used for the image compression is vector quantization (VQ). The Existing methods Linde-Buzo-Gray (LBG) and Fast Back Propagation (FBP) algorithm are presented. In existing methods, the compression ratio is decreased. The proposed method adaptive vector quantization is used to analyze for image vector quantization (VQ). The performance of proposed work is analyzed using the factors SNR, MSE, PSNR and CR. The experimental work using MatLab shows that the proposed scheme is efficient and produced expected result.
\end{abstract}

\section{Keywords}

Vector Quantization, Compression Ratio, Codebook, Image Compression.

\section{INTRODUCTION}

Digital images have turn out to be popular for transferring, sharing store and image in sequence and hence high speed compression technique are need with many advantages of image compression, and the most important one is to reduce the time for the transmission of images. Fundamentally these compression techniques can be categorized into the Lossy compression techniques and lossless compression techniques. The lossy compression technique produces imperceptible difference that may be call visually lossless [3]. Lossless compression is prefer for archival purposes and often for medical imaging, technical drawings, clip art, and comics [16].

\subsection{Compression}

The aim of compression is to decrease the number of bits that are not required to represent data and to decrease the transmission time. Achieve compression by encoding data and the data is decompressed to its original form by decoding. A common compressed file extension is .sit, .tar, .zip; which indicates different types of software used to compress files.

\subsection{Decompression}

The compressed file is initially decompressed and at that time use. There are large numbers of software used to decompress and it depends upon which variety of file is compressed. For example WinZip software is used to decompress .zip file.

\subsection{Vector Quantization}

Vector Quantization is an [3] well-organized method of image compression. VQ compression system contains two components are VQ encoder and decoder. The VQ encoder finds a closest match codeword for each image block in the codebook or directory and the key of the codeword is transmit to VQ decoder. The next phase is decoding phase in which VQ decoder replaces the key values with the relevant codeword from the codebook and produces the quantized image that is called as reconstructed image. A vector quantization is generally defined as a block of pixel values. Vector Quantization is also known as "Block Quantization" or "Pattern Matching Quantization". This process is commonly used in lossy compression methods. It works by programming values from a multidimensional vector space into a finite set of values. A lower-space vector requires less storage space, so the data is compressed. Appropriate to the density matching property of vector quantization, the compressed data contains errors that are inversely comparative to density. Basic working of Vector Quantization is as following:

- Input image.

- Find the closest match code/vector for each image block from the directory or codebook.

- Replaces code /vector by transmit key of code for additional processing.

- $\quad$ Above property is used to reduce the storage space of image.

\subsection{Codebook Design}

The set of quantize output points in VQ is called the codebook of the quantize, and the process of placing these output points is often referred to as codebook design [10]. Assign each element of the training set to the closes representative prototype. After an constituent is assigned, the representative pattern is updated by computing the centroid of the training set vectors assign to it. When the method is complete, we will have $k$ groups of vectors clustered around each of the output points.

A vector is formed using a block of $4 * 4$ pixels forms a vector. For an image size $128 * 128$, the total number of vectors is 1024 out of these every fourth vector is used. This selection is random. Thus, the number of vectors in the initial codebook is 256. $\mathrm{T} 1, \mathrm{~T} 2$, is the input training image. $\mathrm{C} 1, \mathrm{C} 2$, is the codebook. Out of the input vectors, best match is found with the code vectors. The squared distortion is the measure used for this purpose. 


\section{EXISTING METHODOLOGY}

\subsection{LBG Algorithm}

The Linde-Buzo-Gary (LBG) Algorithm starts with the initialization of a codebook which has the random vectors from the training set [1]. Code vectors are generated with the clustering of training set vectors. The centroid of each code is calculated and then interchange with the code vector. This process runs until the distortion in the codebook between iterations reaches a predetermined number. Let $X=(x 1, x 2 \ldots$. $\mathrm{xk})$ be a training vector and $\mathrm{d}(\mathrm{X}, \mathrm{C})$ be the Euclidean distance among any two vectors. The iteration of GLA for a codebook generation is given as follows.

Step 1: Divide the input image into non overlapping blocks and convert each block into vectors.

Step 2: Randomly generate an initial codebook $C B O$

Step 3: $\quad$ Initialize $I=0$.

Step 4: Compute the Euclidean space between all the training vectors belonging to this cluster and the code words in $C B I$. $d X, C=(x t-c t) k 2$ $t=0$.

Step 5: Increment I by one and repeat the step 4 for each code vector.

Step 6: Repeat the Step 3 to Step 5 till codebook of desire size is obtained.

\subsection{Fast Back Propagation Algorithm (FBP)}

In the past years the progress of fast and efficient learning algorithms [2] for ANNs has been a subject of interest. Minimization can be achieved by reducing lemda $(\lambda)$ from unity to zero during the training of the network. The FBP algorithm differs from the original $\mathrm{BP}$ algorithm in the development of the alternative training criterion. This criterion indicates that $\lambda$ must change from 1 to 0 in training phase (i.e. as the total error decreases, should zero). The recommended that the value of should be determined in each adaption cycle from the total error at that point, consequently to some suitable rule $\lambda=\lambda(\mathrm{E})$ where $\mathrm{E}$ is the error of the network. The above indicates that $\lambda=1$ when $E \gg>1$. Here positive integer $n, 1 / E n$ approach zero and, therefore, exp (-1/En) 1. Alternatively, when $E<<1,1 / E n$ becomes very large so $\exp (-1 / E n) 0$. As a result, a proper rule for the reduction of from one to zero is the following Eq.3.1

$$
\lambda=\lambda(E)=\exp (-\mu / E n)
$$

Here a positive real number is $\mu$ and positive integer is $\mathrm{n}$. The lesser the integer $n$, the quicker the reduction of $\lambda$ when $E>>1$.. This is an indication that $\mathrm{n}$ should be greater than 1 . Thus, $\lambda$ is determined in the training of any network according to Eq.3.2
In BPNN guidance by using FBP, use the hyperbolic tangent function instead of sigmoid function used by all the BPNN neurons when using BP. Therefore Eq.3.2 is modified for hyperbolic tangent function as follows:

$$
F^{\prime}(N E T j)
$$

So that F (NETj) lies between -1 and +1 .

Step 1: The FBP algorithm requires, at the initial stage of the training, setting the value of $\lambda$ to 1 .

Step 2: Prior to the BPNN training, the FBP usually $\mu$ lies in the range [0.1-10].

Step 3: The hyperbolic function range of $[-1-+1]$, thus, image normalization is done by [0-255] into the coordinates $[-1-+1]$.

Step 4: The FBP algorithm requires to very small value, usually between [0.01-0.1].

Step 5: the FBP algorithms need value of $\beta$ greater than 1 value of $\beta$ is larger, algorithm is expected to be improved.

Step 6: The output layer neurons in BP algorithm is modified.

Step 7: FBP algorithm, is calculated in each iteration for the purpose of $\lambda$ adaptation.

\subsection{Drawbacks of Existing System}

- Codebook formulation is also requires more time.

- While LBG is efficient and very good choice for data transmission as its uses minimum codebook entries.

- To compare those parameter in order to achieve low SNR, MSE, PSNR and high CR.

- $\quad$ LBG algorithm designs very large codebook and performance is also not good.

- It requires more storage space for the codebook.

- It has a complete design requires a large number of computations.

- It convergence time is very large.

\section{PROPOSED METHODOLOGY}

The main objective of this proposed system to improve the quality of the image and reduce the noise. In the proposed approach the vector quantization is applied to compression using the AVQ to improve the quality of the image, reduce the noise of the input image and achieve good compression ratio. 


\section{AVQ Encoder Algorithm}

Step 1: Initialize the dictionary $D$ to all the possible values of the image pixels.

Step 2: Initialize the pool of growing points $(G P P)$ to one or more growing point $G$ from $G P P$.

Step 3: Repeat Step 3-7 until the GPP is empty.

Step 4: Use a growing algorithm to select a growing point $G$ from $G P P$.

Step 5: Grow an image block $B$ with $G$ as its corner.

Step 6: Use a matching algorithm to match $B$, as it is being grown, to a dictionary entry.

Step 7: Once B has reached the maximum size where it still can be matched depends on the size of $D$.

Step 8: Delete $G$ from the GPP and use an algorithm which new growing points to add to the GPP.

Step 9: If D is full, use an algorithm to delete one or more entries. Update the dictionary based on $B$.

\section{AVQ Decoder Algorithm}

Step 1: Initialize the dictionary $D$ and the GPP as in Step 1 of the encoder.

Step 2: Repeat Step 3-7 until the GPP is empty.

Step 3: Use the encoder's growing algorithm to select a growing point $G$ from the GPP.

Step 4: Input a pointer from the compressed stream, use it to retrieve a dictionary entry $d$, and place $d$ at the location and position specified by $G$.

Step 5: Delete G from the GPP and use an algorithm which new growing points to add to the GPP.

Step 6: If D is full, use an algorithm to delete one or more entries.

Step 7: Update D and the GPP as in Step 6-7 of the encoder.

\section{EXPERIMENTS AND RESULTS}

\subsection{Performance Metrics}

The performance of a proposed algorithm must be evaluated by considering different objective such as Signal to Noise Ratio (SNR), Mean Squared Error (MSE), Peak Signal to Noise Ratio (PSNR) and Compression Ratio (CR) are most commonly used as a measure of quality of image compression.

Table 4.1 Performance Analysis for SNR Value

\begin{tabular}{|l|c|c|c|}
\hline Methods/Images & LBG & FBP & AVQ \\
\hline Cameraman & 0.2678 & 0.2950 & 0.3024 \\
\hline Vegetables & 0.2785 & 0.3050 & 0.3105 \\
\hline Mandi & 0.2345 & 0.2990 & 0.3215 \\
\hline
\end{tabular}

Table4.2 Performance Analysis for MSE Value

\begin{tabular}{|l|c|c|c|}
\hline \multicolumn{1}{|c|}{ Methods/Images } & LBG & FBP & AVQ \\
\hline Cameraman & 0.06 & 0.07 & 0.05 \\
\hline Vegetables & 0.08 & 0.05 & 0.04 \\
\hline Mandi & 0.13 & 0.11 & 0.09 \\
\hline
\end{tabular}

Table 4.3 Performance Analysis for PSNR Value

\begin{tabular}{|l|c|c|c|}
\hline \multicolumn{1}{|c|}{ Methods/Images } & LBG & FBP & AVQ \\
\hline Cameraman & 18.7 & 20.5 & 32 \\
\hline Vegetables & 15.7 & 29.0 & 35 \\
\hline Mandi & 32.5 & 35.1 & 39 \\
\hline
\end{tabular}

Table 4.4 Performance Analysis for CR Value

\begin{tabular}{|l|c|c|c|}
\hline \multicolumn{1}{|c|}{ Methods/Images } & LBG & FBP & AVQ \\
\hline Cameraman & 1.85 & 1.78 & 1.56 \\
\hline Vegetables & 1.46 & 1.35 & 0.78 \\
\hline Mandi & 1.67 & 1.60 & 0.39 \\
\hline
\end{tabular}

\section{CONCLUSION}

Image compression techniques are becoming very vital role in the area of image analysis, analysis statistical, analysis text, data mining, web mining etc. The image compression in terms of compression ratio and compression size. The proposed algorithm achieves lower bits rate at the same image quality. After performing different images it is concluded that proposed AVQ algorithm is the best one in all compression ratio parameters. The proposed work is compared with other existing method which produced better results for image compression.

In the future, we will consider combining various neural network methods into current system. It can be considered in the future to provide more accurate results in the image compression. In image compression future to provide the effective enhancement of the image.

\section{REFERENCES}

[1] Abdelatief. H Abouali, "Image Compression Using Adaptive LBG'International Journal of Emerging Trends \& Technology in Computer Science (IJETTCS), Volume 2, Issue 4, July - August 2013 ISSN 2278-6856.

[2] Al-Allaf, O.N.A., "Fast BackPropagation Neural Network algorithm for reducing convergence time of BPNN Image Compression", Information Technology and Multimedia (ICIM), Nov. 2011.

[3] Amrutbhai N Patel , Dr .D. J. Shah ,"Performance Analysis Of Vector Quantization Based Lossy Image Compression", Journal Of Information, Knowledge And Research In Electronics And Communication Nov 14 To Oct 15 |Volume - 03, Issue - 02 .

[4] Arup Kumar Pal and AnupSar, "An Efficient Codebook Initialization Approach For LBG Algorithm", International Journal of Computer Science, Engineering and Applications (IJCSEA) Vol.1, No.4, August 2011. 
[5] Mrs.BhumikaGupta,PauriGarhwalUttrakhand,"Study of Various Lossless Image Compression Technique ", International Journal of Soft Computing and Engineering (IJSCE) ISSN: 2231-2307, Volume-2, Issue-3, July 2012.

[6] Tzu-Chuen Lu, Ching-Yun Chang "A Survey of VQ Codebook Generation"Journal of Information Hiding and Multimedia Signal ProcessingUbiquitous InternationalVolume 1, Number 3, July 2010.

[7] Dinesh V. Rojatkar, Nitesh D. Borkar, Buddhabhushan R. Naik, RavindraN. Peddiwar, "Image Compression Techniques: Lossy and Lossless", InternationalJournal of Engineering Research and General Science Volume 3, Issue 2, March- April, 2015.

[8] ChetanDudhagara, Dr. KishorAtkotiya, "Image Compression using Vector Quantization", International Journal of IT, Engineering and Applied Sciences Research (IJIEASR) ISSN: 2319-4413 Volume 2, No. 2, February2013.

[9] Dr.BEswara Reddy and K. VenkataNarayana,"A Lossless Image Compression Using Traditional And Lifting Based Wavelets", Signal \& Image Processing : An International Journal (SIPIJ) Vol.3, No.2, April 2012.

[10] Dr.S.Vimala, Ms.S.Ezilarasi, "Classified Codebook with Indexmap Compression for Vector Quantization to Compress still Images" Volume: 5 Issue : 4, April 2015.

[11] Mary Jansi Rani. Y, Pon. L.T. Thai, John Peter. K,"Visually Lossless Compression for Color Images with Low Memory Requirement using Lossless Quantization", International Journal of Soft Computing and Engineering (IJSCE), ISSN: 2231-2307, Volume-2, Issue-3, July 2012.

[12] Dr. H. B. Kekre, Tanuja K. Sarode, "New Clustering Algorithm for Vector Quantization using Rotation of Error Vector", International Journal of Computer Science and Information Security, Vol. 7, No. 3, 2010.

[13] H.B. Kekre, T. Sarode\& P. Natu, “ Image Compression using Fusion of Hybrid Wavelet Transform andVector Quantization",African Journal of Computing \& ICT Reference Format, Vol 7, No. 5. Pp 85-94,December 2014.

[14] Y. Linde, A. Buzo, and R. M. Gray, "An Algorithm for Vector Quantizer Design", IEEE Transactions on Communications, pp. 702-710, January 1980.

[15] Mukesh Mittal, RuchikaLamba, "Image Compression Using Vector Quantization Algorithms: A Review", International Journal of Advanced Research in Computer Science and Software Engineering (IJARCSSE), Volume 3, Issue 6, June 2013.

[16] MalwinderKaur, NavdeepKaur, "A Literature Survey On Lossless Image Compression", International Journal of Advanced Research in Computer and Communication Engineering, Vol. 4, Issue 3, March 2015.
[17] NirbhayKashyap, Dr. Shailendra Narayan Singh "Review of Image Compression and Comparison of its Algorithms" International Journal of Application or Innovation in Engineering \& Management (IJAIEM) Volume 2, Issue 12, December 2013.

[18] S. S. Panda, M.S.R.S Prasad, MNM Prasad, Ch. SKVR Naidu, "Image Compression Using Back Propagation Neural Network",International Journal of Engineering Science \& Advanced Technology Volume - 2, Issue - 1, 74 - 78 Jan- Feb 2012.

[19] N. M. Nasrabadi and YushuFeng "Image Compression using address vector Quantization "IEEE Trans. On Communications, Vol.38.No.2 Dec.1990, pp 2166-2173.

[20] SarangBansod, Shweta Jain "Recent Image Compression Algorithms: A Survey” International Journal of Advanced Research in Computer and Communication Engineering (IJARCCE) Vol.2, Issue 12, December 2013.

[21] P.Sivakumar, S.Ravi, "Vector Quantization Based Image Compression", International Journal of Innovative Technology and Exploring Engineering (IJTTEE) ISSN: 2278.-3075, Volume-1, Issue-1, June 2012.

[22] Syed Aseem Ahamed1, K Chandrashekarappa, "ANN Implementation for Image Compression and Decompression Using Back Propagation Technique", International Journal of Science and Research (IJSR) Volume 3 Issue 6, June 2014.

\section{AUTHOR PROFILE}

A.Divya received Bachelor of Computer Science (B.Sc.) degree from the Bharathiar University, in 2012 and the Master of Computer Science (M.Sc) degree from the Bharathidasan University, Thirullapallai in 2014. She also recieved the M.Phil degree from the Bharthiar Unviersity, Coimbatore, in 2015. Her research area includes Digital Image Processing.

Dr. S. Sukumaran graduated in 1985 with a degree in Science. He obtained his Master Degree in Science and M.Phil in Computer Science from the Bharathiar University. $\mathrm{He}$ received the $\mathrm{Ph} . \mathrm{D}$ degree in Computer Science from the Bharathiar University. He has 27 years of teaching experience starting from Lecturer to Associate Professor. At present he is working as Associate Professor of Computer Science in Erode Arts and Science College, Erode, Tamil nadu. He has guided for more than 50 M.Phil research Scholars in various fields and guided 5 Ph.D Scholars. Currently he is Guiding 5 M.Phil Scholars and 6 Ph.D Scholars. He is member of Board studies of various Autonomous Colleges and Universities. He published around 50 research papers in national and international journals and conferences. His current research interests include Image processing, Network Security and Data Mining. 